\title{
BER Analysis in Multi Carrier OFDM Scheme For 5G Wireless Communications
}

\author{
Vemakoti Vivekanand, Sathish Kumar Yegireddi
}

\begin{abstract}
The statistic shows the number of mobile phone users in India from 2013 to 2019. For 2017 the number of mobile phone users in India is expected to rise to $\mathbf{7 3 0 . 7}$ million. In this same year the number of smart phone users in India is predicted to reach 340 million and could reach almost 468 million by 2021.The spectacular growth of wireless communication tools has escalated the number of mobile subscribers from almost 700 million in 2000 to more than 4 billion in 2009. The huge number of subscribers has led to several issues with how service is provided. The high user demand has forced developers to overcome the problems of the old analog systems and to introduce OFDM as a promising technique that can fulfill users' high demands. This technique matches well with high data rate connection and provides a higher capacity for the subscribers' usage. The OFDM, as a multi-carrier, is more complex than the single-carrier transmission scheme. However, the OFDM technique maintains better performance for high data rate in terms of bit error rate (BER). Here in this report we have developed a MATLAB simulink model using OFDM technique for analyzing SNR and BER of a randomly generated bit stream.
\end{abstract}

Index Terms - BER, OFDM, SNR

\section{INTRODUCTION}

In telecommunications, frequency-division multiplexing (FDM) is a technique by which the total bandwidth available in a communication medium is divided into a series of non-overlapping frequency bands, each of which is used to carry a separate signal. This allows a single transmission medium such as a cable or optical fiber to be shared by multiple independent signals. Another use is to carry separate serial bits or segments of a higher rate signal in parallel.

The most natural example of frequency-division multiplexing is radio and television broadcasting, in which multiple radio signals at different frequencies pass through the air at the same time. Another example is cable television, in which many television channels are carried simultaneously on a single cable. FDM is also used by telephone systems to transmit multiple telephone calls through high capacity trunk lines , communications satellites to transmit multiple channels of data on uplink and downlink radio beams, and broadband DSL modems to transmit large amounts of computer data through twisted pair telephone lines, among many other uses.

An analogous technique called wavelength division multiplexing is used in fiber-optic communication, in which

Vemakoti Viveknand M.Tech, MBA, is a curious researcher in quantum electrodynamics and high speed signal transmissions though had a deep insights on photovoltaic cells' performance calibrations in his previous research papers.

Sathish Kumar Yegireddi M.Tech (Embedded System)

Department of ECE,IIIT Srikakulam, RAJIV GANDHI UNIVERSITY OF KNOWLEDGE TECHNOLOGIES(RGUKT),Andhra Pradesh, India. multiple channels of data are transmitted over a single optical fiber using different wavelengths (frequencies) of light.

\section{ORTHOGONAL FREQUENCY DIVISION MULTIPLEXING}

\section{A. Introduction}

Frequency division multiplexing:

In telecommunications, frequency-division multiplexing (FDM) is a technique by which the total bandwidth available in a communication medium is divided into a series of non-overlapping frequency bands, each of which is used to carry a separate signal. This allows a single transmission medium such as a cable or optical fiber to be shared by multiple independent signals. Another use is to carry separate serial bits or segments of a higher rate signal in parallel.

The most natural example of frequency-division multiplexing is radio and television broadcasting, in which multiple radio signals at different frequencies pass through the air at the same time. Another example is cable television, in which many television channels are carried simultaneously on a single cable. FDM is also used by telephone systems to transmit multiple telephone calls through high capacity trunk lines , communications satellites to transmit multiple channels of data on uplink and downlink radio beams, and broadband DSL modems to transmit large amounts of computer data through twisted pair telephone lines, among many other uses.

An analogous technique called wavelength division multiplexing is used in fiber-optic communication, in which multiple channels of data are transmitted over a single optical fiber using different wavelengths (frequencies) of light.

OFDM:

In telecommunications, orthogonal frequency-division multiplexing (OFDM) is a method of encoding digital data on multiple carrier frequencies. OFDM has developed into a popular scheme for wideband digital communication, used in applications such as digital television and audio broadcasting, DSL internet access, wireless networks, power line networks, and $4 \mathrm{G}$ mobile communications.

OFDM is a frequency-division multiplexing (FDM) scheme used as a digital multi-carrier modulation method. OFDM was introduced by Chang of Bell Labs in 1966.Numerous closely spaced orthogonal sub-carrier signals with overlapping spectra are emitted to carry data Demodulation is based on Fast Fourier Transform algorithms. OFDM was improved by Weinstein and Ebert in 1971 with the introduction of a guard interval, providing better orthogonality in transmission channels affected by multipath propagation. Each sub-carrier (signal) is modulated with a conventional modulation scheme . without complex 
equalization filters. Channel equalization is simplified because OFDM may be viewed as using many slowly modulated narrowband signals rather than one rapidly modulated wideband signal. The low symbol rate makes the use of a guard interval between symbols affordable, making it possible to eliminate inter symbol interference (ISI) and use echoes and time-spreading (in analog television visible as ghosting and blurring, respectively) to achieve a diversity gain, i.e. a signal-to-noise ratio improvement. This mechanism also facilitates the design of single frequency networks (SFNs) where several adjacent transmitters send the same signal simultaneously at the same frequency, as the signals from multiple distant transmitters may be re-combined constructively, sparing interference of a traditional single-carrier system.

\section{NEED FOR OFDM}

Orthogonal Frequency Division Multiplexing (OFDM) is a technique for transmitting large amounts of digital data over a radio wave the technology works by splitting the radio signal into multiple smaller sub-signals that are then transmitted simultaneously at different frequencies to the receiver.

\section{MULTIPATH FADING}

It occurs when a transmitted signal divides and takes more than one path to a receiver and some of the signals arrive out of phase, resulting in a weak or fading signal. Some TRANSMISSION LOSSES that affect radio-wave propagation are ionospheric absorption, ground reflection, and free-space losses.

Multipath fading occurs when the receiver antenna receives different signals from different paths Multipath fading could be formed in many ways by various causes as shown in Figure 1. In the ideal situation, the signal should propagate directly to the antenna with no obstacles. However, in real applications, natural huge objects such as a mountain could cause shadowing or scattering of the signal due to the mountain's rough surface. The signal could also be reflected or diffracted because of buildings or trees

\section{B. Idealized System Model}

This section describes a simple idealized OFDM system model suitable for a time-invariant AWGN channel.

\section{TRANSMITTER:}

An OFDM carrier signal is the sum of a number of orthogonal sub-carriers, with baseband data on each sub-carrier being independently modulated commonly using some type of quadrature amplitude modulation (QAM) or phase-shift keying (PSK). This composite baseband signal is typically used to modulate a main RF carrier. A serial stream of binary digits.By inverse multiplexing, these are first demultiplexed into parallel streams, and each one mapped to a (possibly complex) symbol stream using some modulation constellation (QAM, PSK, etc.). Note that the constellations may be different, so some streams may carry a higher bit-rate than others.
An inverse FFT is computed on each set of symbols, giving a set of complex time-domain samples. These samples are then quadrature-mixed to passband in the standard way. The real and imaginary components are first converted to the analogue domain using digital-to-analogue converters (DACs); the analogue signals are then used to modulate cosine and sine waves at the carrier frequency, respectively. These signals are then summed to give the transmission signal.

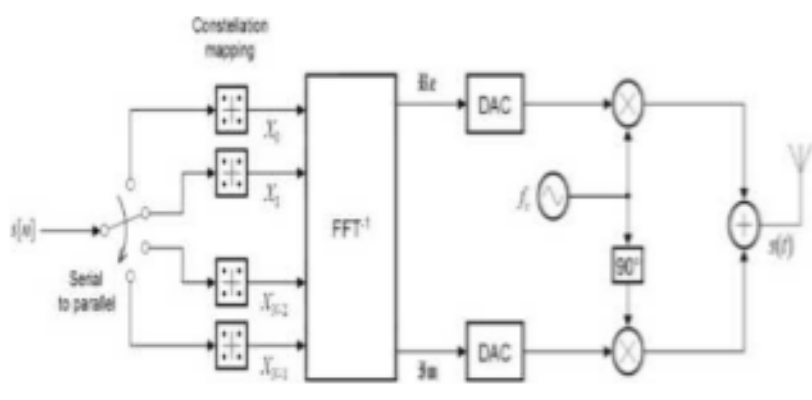

Figure 1.Idealized System Model Transmitter Section

\section{RECEIVER:}

The receiver picks up the signal, which is then quadrature-mixed down to baseband using cosine and sine waves at the carrier frequency. This also creates signals centered on so low-pass filters are used to reject these. The baseband signals are then sampled and digitised using analog-to- digital converters (ADCs), and a forward FFT is used to convert back to the frequency domain. This returns parallel streams, each of which is converted to a binary stream using an appropriate symbol detector. These streams are then re-combined into a serial stream, which is an estimate of the original binary stream at the transmitter.

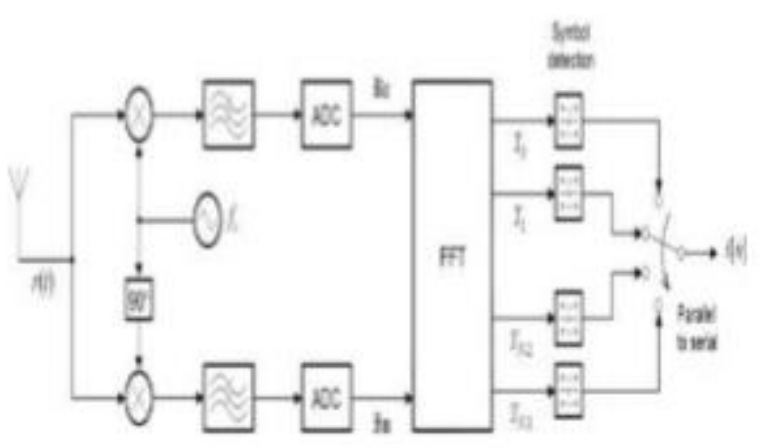

Figure 2. Block Diagram Of Idealized Model Receiver Section

\section{ADVANTAGES}

Immunity to selective fading:

One of the main advantages of OFDM is that is more resistant to frequency selective fading than single carrier systems because it divides the overall channel into multiple narrowband signals that are affected individually as flat fading sub-channels. 
Equalization is very simple compared to single carrier systems.

\section{APPLICATIONS:}

OFDM has developed into a popular scheme for wideband digital communication, used in applications such as

- digital television

- audio broadcasting

- DSL internet access

- wireless networks

- power line networks

- $4 \mathrm{G}$ mobile communications

\section{WAVEFORM FOR OFDM:}

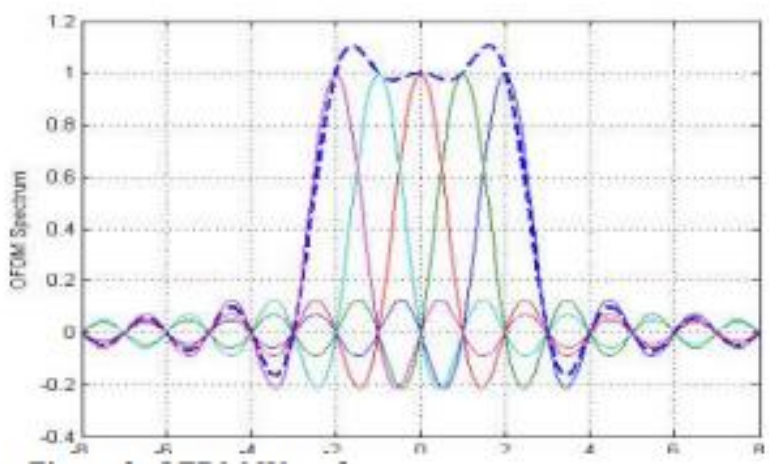

Figure 3. OFDM Waveform

\section{SISO-OFDM Functional Block Diagram}

Figure depicts the various functional blocks of SISO-OFDM link. In SISO, the information bits are generated, complex symbol mapping is done and inverse fast Fourier transform is taken to convert frequency domain signals into OFDM time domain signals. Cyclic prefix is added to overcome interference between subcarriers and parallel to serial conversion takes place and then it is transmitted via single antenna which operates at $2.4 \mathrm{GHz}$.

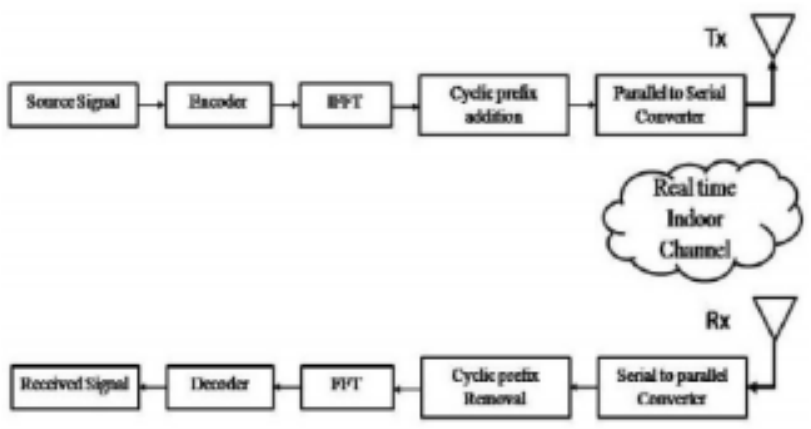

Figure 4. SISO-OFDM Functional Block Diagram

\section{MIMO-OFDM Functional Block Diagram}

It is the dominant air interface for $4 \mathrm{G}$ and $5 \mathrm{G}$ broadband wireless communications. It combines multiple-input, multiple-output (MIMO) technology, which multiplies capacity by transmitting different signals over multiple antennas, and orthogonal frequency-division multiplexing (OFDM), which divides a radio channel into a large number of closely spaced subchannels to provide more reliable communications at high speeds. Research conducted during the mid-1990s showed that while MIMO can be used with other popular air interfaces such as time-division multiple access (TDMA) and code-division multiple access (CDMA), the combination of MIMO and OFDM is most practical at higher data rates.

\section{E. Conventional MIMO-OFDM System}

The general structure of MIMO-OFDM system is shown in figure 1 . The proposed system consists of 2 transmit and 2 receive antennae. The OFDM signal for each antenna is obtained by applying the inverse Fast Fourier transform (IFFT) and can be detected using Fast Fourier transform (FFT) [5]. A pilot sequence is inserted and used for the channel estimation. Also, a cyclic prefix is inserted in front of the OFDM symbol at the last step of OFDM modulation block. The time length of the cyclic prefix should be greater than the maximum delay spread of the channel. The main function of the cyclic prefix is to guard the OFDM symbol against Inter Symbol Interference (ISI), hence, this cyclic prefix is called the guard interval of the

OFDM symbols [Ref]. The MIMO coding can use several encoders such as STBC, VBLAST and Golden coding. In this paper, the conventional MIMO-OFDM system is implemented using Altamonte STBC with two transmits and two receive antennas.

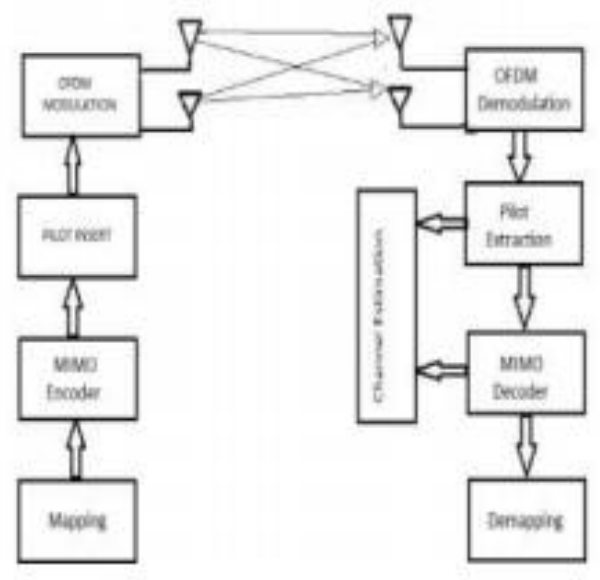

Figure 5. Conventional MIMO-OFDM System

\section{F. New Transmission model}

The new transmission model is suitable for symmetric channels, such as the transmission between two base stations, microwave links, or radio beam transmission. The proposed MIMO- OFDM model is shown in the following figure 


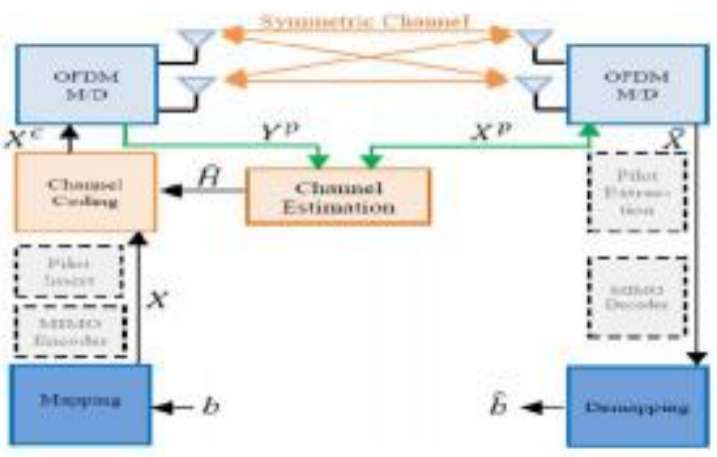

Figure 6. MIMO-OFDM

This new MIMO-OFDM model, the channel parameters are estimated from a pilot data transmitted by the receiver end. These estimated parameters are used by a special channel coding block to adapt the transmitter signal to the diverse channel impairments and variations. To reduce the system complexity we have removed the pilot insert, the pilot extraction, the MIMO encoder and the MIMO decoder from the conventional MIMO-OFDM scheme. The channel coding is based on the channel variations, this channel in our case is between two transmit antennae and two receive antennae, and it can be modelled as shown in the figure 2 . First, the receiver send a pilot signal to the transmitter.

\section{G. Simulation Results}

The proposed MIMO-OFDM transceiver system is simulated using parameters shown in Table. These parameters are based on transmission between two base stations in LTE system

\begin{tabular}{|l|l|}
\hline $\begin{array}{l}\text { PARAMETERS } \\
\text { SPECIFOCATIONS }\end{array}$ & \\
\hline SYSTEM MIMO-OFDM & MIMO-OFDM \\
\hline CONSTELLATION 16 -QAM & 16 -QAM \\
\hline$T_{S}(\mu S)$ & 72 \\
\hline$f_{c}(G H Z)$ & 2.15 \\
\hline$\delta f(K H Z)$ & 15 \\
\hline$B(M H Z)$ & 5 \\
\hline SIZE OF DFT/IDFT & 512 \\
\hline & $\begin{array}{l}\text { ALTAMONTE STBC (2TX } \\
\text { 2RX) }\end{array}$ \\
\hline MIMO ENCODER & \\
\hline
\end{tabular}

\section{TABULAR 1. SIMULATION RESULTS}

In this part we are interested in comparing the proposed scheme with the conventional MIMO- OFDMof this simulation considers the transmission between two base stations in LTE system. The channel parameters are used for the channel coding in the new proposed scheme.Figure 5 shows the variation of BER as a function of Es/NO. The proposed scheme has better performances than the standard MIMO-OFDM system. Besides the performance of this scheme, its complexity is low and there are no needs of complicate MIMO encoder or channel estimation at the reception. In addition, to the simplification of the conventional MIMO-OFDM transceiver structure, bandwidth efficiency (also frame efficiency) can be highly increased.

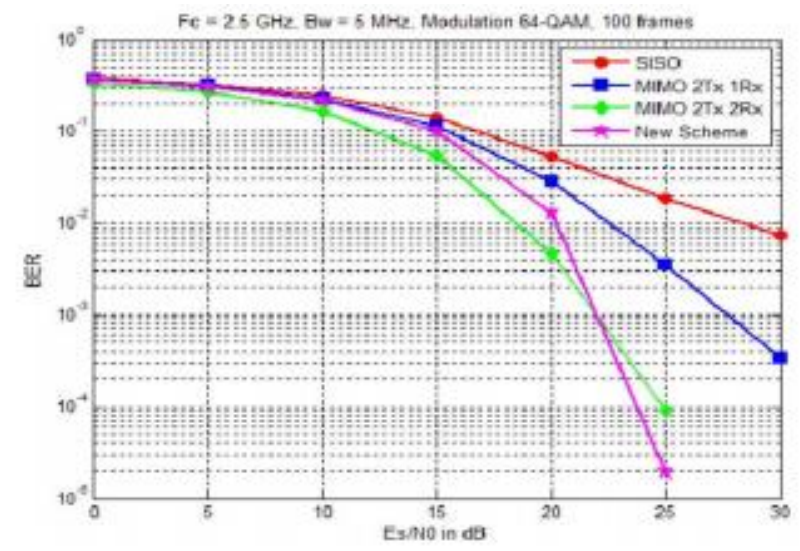

FigURE 7. BIT ERROR RATE AS A FUNCTION OF SIGNAL TO NOISE RATIO

As an example, assume this transmitted bit sequence: 011 0001011 and the following

received bit sequence: 0010101001 , The number of bit errors (the underlined bits) is, in this case, 3. The BER is 3 incorrect bits divided by 10 transferred bits, resulting in a BER of 0.3 or $30 \%$

\section{Overview}

This contribution introduces a new transmission scheme for multiple-input multiple-output (MIMO) orthogonal frequency division multiplexing (OFDM) systems. This scheme is based on Channel coding using estimated channel parameters from a transmitted pilot data at the receiver end. Consequently, the prior information used by the coding scheme, will help the transmitted signal to adapt to the channel impairments and be more resilient to noise and interference. Simulation results confirm the high performance and the low complexity of the proposed scheme when compared to the conventional MIMOOFDM system using Altamonte STBC Coding

\section{PROPOSED CHANNELS}

\section{A. Additive White Gaussian Noise (AWGN)}

\section{1) Introduction:}

Additive White Gaussian Noise (AWGN) is common to every communication channels, which is the statistically random radio noise characterized by a wide frequency range with 
regards to a signal in the communications channel. This assignment describes two aspects of telecommunications engineering: i) the basic understanding of MATLAB, and ii) the effect of Additive white Gaussian Noise (AWGN) on the transmitted data using based-band simulation under different values of signal-to-noise (SNR) ratio with this tool. In my experiment, I use MATLAB tools to generate the noise sequence, the data sequence, the both data and noise sequence and add them together, recover signal from noisy received data and calculate noise power, calculate SNR and BER and then plot them with different SNR values.

The random nature of noise in time domain will, on occasions, cause a transmitted symbol to be distorted such that the receiver interprets it as a different symbol in the modulation scheme alphabet. Under these circumstances, a given average level of AWGN introduces an average number of symbol errors where each symbol error causes one or more bit errors at output of the receiver. The errors may be characterized by a bit error rate (BER).

\section{2) Important Terminologies}

Additive white gaussian noise (awgn):

A basic and generally accepted model for thermal noise in communication channels, is the set of assumptions that the noise is additive, i.e., the received signal equals the transmit signal plus some noise, wherethe noise is statistically independent of the signal. the noise is white, i.e, the power spectral density is flat, so the autocorrelation of the noise in time domain is zero for any non-zero time offset. the noise samples have a Gaussian distribution. Additive White Gaussian Noise (AWGN) is the statistically random radio noise characterized by a wide frequency range with regards to a signal in a communications channel.

\section{Average Signal-to-Noise Ratio (SNR):}

Signal-to-noise ratio is a term for the power ratio between a signal (meaningful information) and the background noise

$$
\begin{gathered}
\mathrm{SNR}=\frac{P_{s}}{P_{n}}=\left|\frac{A_{n}}{A_{n}}\right|^{2} \\
\mathrm{SNR}(\mathrm{dB})=10 \log \frac{P_{s}}{P_{n}}-20 \log \frac{A_{n}}{A_{n}} \mid
\end{gathered}
$$

Bit Error Rate (BER):

Bit error ratio (BER) is the number of bits received in error, divided by the total number of bits. Following are Wave forms

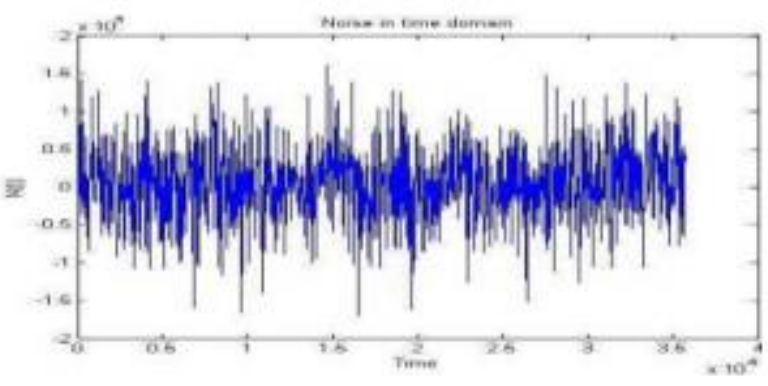

FIGURE 8. Wave Form Of Noise (AWGN) in time domain

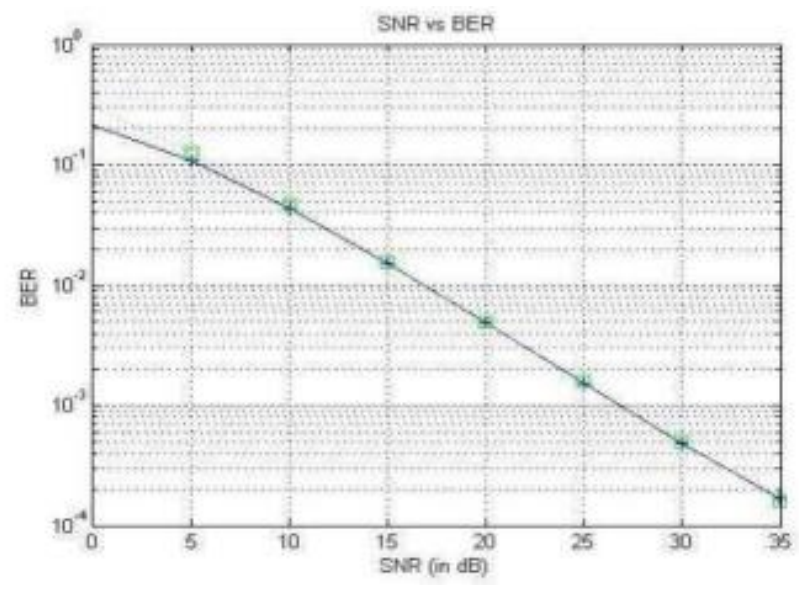

FIGURE 9.Graph of SNR vs BER for different SNR values.

From the above graph BER goes down for increased signal power (Blue line) and the green line shows the SNR versus Estimated values of Bit Error probability which also follows the same trends. The effect of Additive White Gaussian Noise on transmitted data. From the above analysis, SNR can improve while the signal power is more than the noise power.

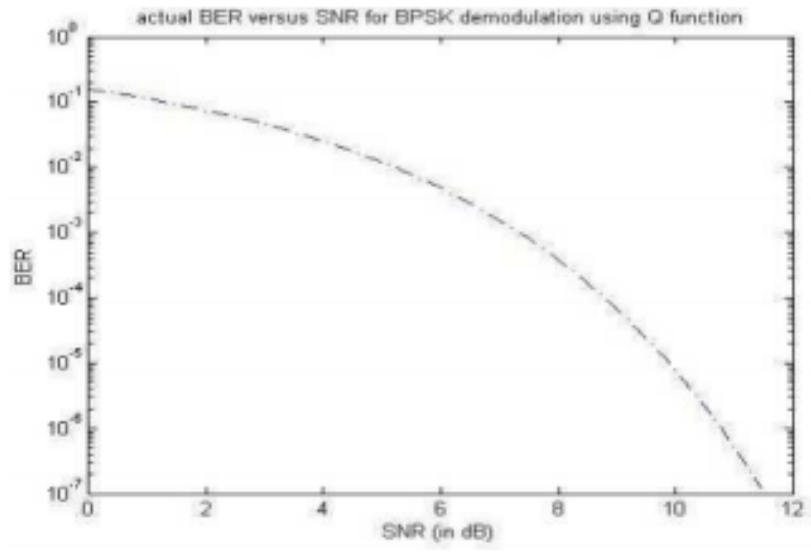

FIGURE 10. Graph of Actual SNR vs BER for BPSK Demodulation

We see the effect of AWGN for both BPSK and QPSK modulation techniques. In both cases, we conclude the same thing as above.

\section{B. Nakagami Fading Channel Distribution}

The Nakagami distribution is relatively new, being first proposed in 1960.It has been used to model attenuation of wireless signals traversing multiple paths and to study the impact of fading channels on wireless communications.

The Nakagami distribution or the Nakagami-m distribution is a probability distribution related to the gamma distribution. The family of Nakagami distributions has two parameters: a shape parameter and a second parameter controlling spread. 
Based on the approximated expression of the Q-function by Chiani, Dardari, and Simon, a simple and accurate generalized closed-form expression for the bit error rate (BER) over Nakagami-m fading channel is derived. The expression is given as a product of the well-known gamma function and a finite sum of functions of the Nakagami $-\mathrm{m}$ fading parameter. The derived expression is applicable for any real $\geq 0.5$, and for any coherent modulation scheme of any order. Numerical results are used to validate the derived expressions using different combinations of modulation orders and Nakagami-m fading parameters.

\section{1) Introduction}

Bit Error Rate (BER) analysis of wireless communication systems over fading channels is an important performance metric to measure the quality of transmission (i.e., quality of service) over fading channels. A crucial finding that many researchers are trying to achieve is having a closed form expression that can evaluate the BER performance as function of the wireless channel statistics and the system parameters (e.g., modulation order). Such closed-form expressions will enable designing, implementing and testing of wireless systems in an optimum way.

The BER analysis over Nakagami-m fading channels requires evaluation of integral that involves the well-known Q-function. The Q-function itself is an integral that cannot be reduced to closed-form, but instead is tabulated. In the literature, there are many researchers who tried to find an approximated expression for the Q-function. These approximations differ in its accuracy and complexity. In order to carry out analytical formulation of many cases involving digital transmission over Nakagami-m Fading channel, researchers need to use a simple yet accurate expression for such cases, and generalize the analysis to involve all kind of modulations (i.e., coherent and non-coherent). the analysis over Nakagami-m fading channels but their derived expressions lacks simplicity. an expression for Binary Phase Shift Keying (BPSK) but it lacks generality as well as simplicity.

the Symbol Error Rate (and hence BER) performance over Nakagami-m fading channels. This expression is based on the Q-function. The accuracy of the derived expression is very high and it can be used for any value of fading index $m$ and for any digital modulation scheme of any modulation order. The derived expression has no limitations in terms of the range of average signal-to-noise ratio. Noteworthy, the expression is given as a product of the well-known gamma functions and a finite sum of functions of the Nakagami-m fading parameter.

\section{M or $\mu \geq 0.5$ shape(real),}

\section{$\Omega$ or $w \geq 0$ spread(real),}

\section{Simulation Result}

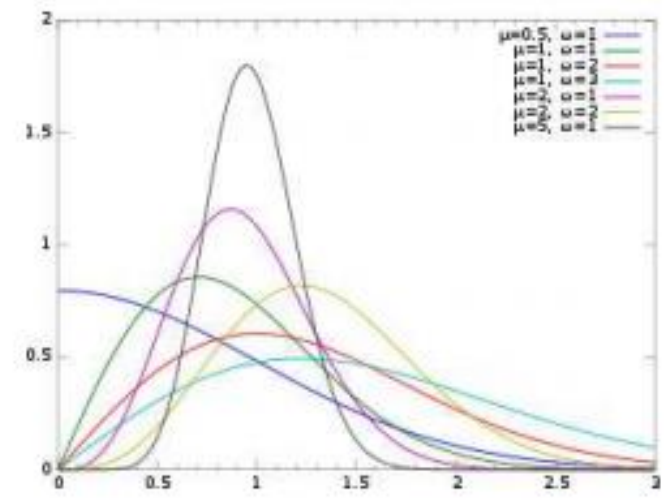

FIGURE 11. Wave forms Of Distributed Channel

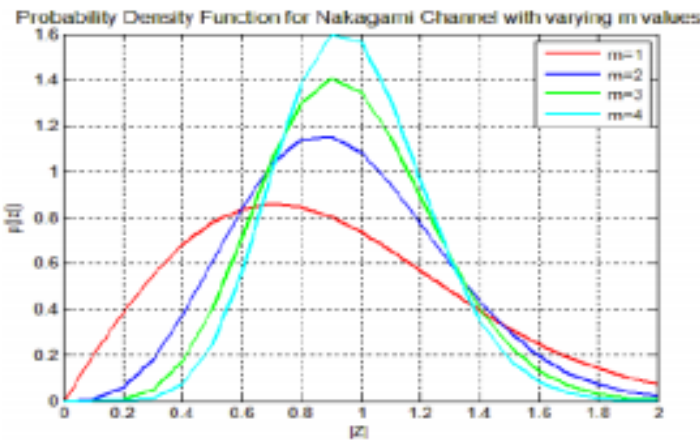

FIGURE 12.Waveform of PDF with varying $m$ values

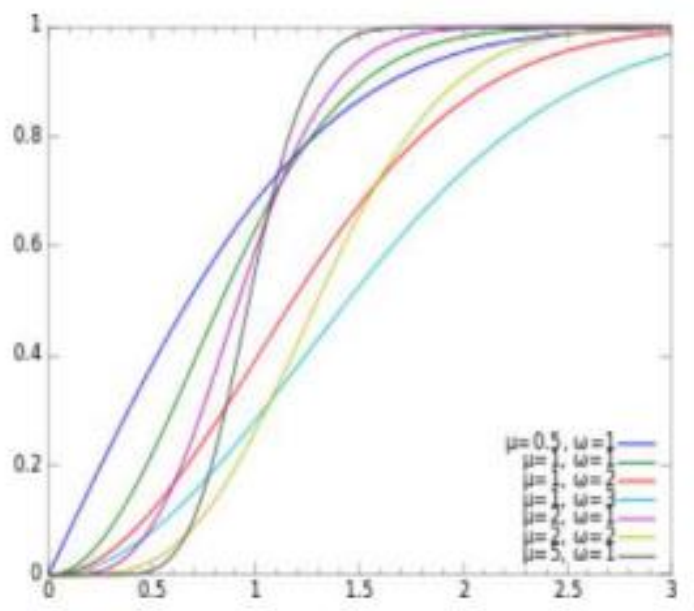

FIGURE 13. Graph of Cumulative Distribution Function

\section{PRAPOSED CODING TECHNICS}

\section{A. Channel coding}

Channel coding, also known as forward error control coding (FECC), is a process of detecting and correcting bit errors in digital communication systems. Channel coding is performed both at the transmitter and at the receiver. At the transmit side, channel coding is referred to as encoder, where extra bits (parity bits) are added with the raw data before modulation. At the receive side, channel coding is referred to as the decoder. Channel coding enables the receiver to detect and correct errors, if they occur during transmission due to noise, interference and fading. 
- channel coding techniques for wireless communications such as

- linear convolution codes

- turbo codes,

- low parity check codes (LDPC)

- space-time coding,

- Reed Solomon (RS) codes

- convolutional codes.

- LT codes.

\section{B. Source coding techniques:}

Entropy of a source is the measure of information. Basically, source codes try to reduce the redundancy present in the source, and represent the source with fewer bits that carry more information.

$C(x) \geq H(x)$, where $H(x)$ is entropy of source (bitrate), and $C(x)$ is the bitrate after compression.

- Huffman Code.

- Lemple-Ziv Code.

- Shannon fanon Code.

- Arithmetic Code.

\section{Gray code}

Gray codes are another numerical code representation in which consecutive data differ in only one bit like 000, 001, 011, 010, 110, 111, soon.

Gray codes have application in $\mathrm{ADC}$ (Analog to Digital Converter) designing, they are used to encode the position of a motor, let's divide a circle into 8 sectors and then divide it into 3 concentric circles, each sector is representing one location of the motor and word length is 3 bits $\left(2^{\wedge} 3=8\right)$.

- detect single bit coding errors.

- In rotary systems, single-track Gray Codes (STGC) are used to sense contacts with rotary tracks.

\section{BER}

The bit error rate (BER) is the number of bit errors per unit time. The bit error ratio (also BER) is the number of bit errors divided by the total number of transferred bits during a studied time interval. Bit error ratio is a unitless performance measure, often expressed as a percentage. $\mathrm{BER}$ is

\section{Errors}

\section{Total Number of BIts}

\section{A. Factors Effecting BER:}

It can be seen from using $\mathrm{E}_{b} / \mathrm{N}_{\mathrm{o}}$, that the bit error rate, BER can be affected by a number of factors. By manipulating the variables that can be controlled it is possible to optimise a system to provide the performance levels that are required. This is normally undertaken in the design stages of a data transmission system so that the performance parameters can be adjusted at the initial design concept stages

Interference: The interference levels present in a system are generally set by external factors and cannot be changed by the system design. However it is possible to set the bandwidth of the system. By reducing the bandwidth the level of interference can be reduced. However reducing the bandwidth limits the data throughput that can be achieved.

Increase transmitter power: It is also possible to increase the power level of the system so that the power per bit is increased. This has to be balanced against factors including the interference levels to other users and the impact of increasing the power output on the size of the power amplifier and overall power consumption and battery life, etc.

Reduce bandwidth: Another approach that can be adopted to reduce the bit error rate is to reduce the bandwidth. Lower levels of noise will be received and therefore the signal to noise ratio will improve. Again this results in a reduction of the data throughput attainable.

Lower order modulation: Lower order modulation schemes can be used, but this is at the expense of data throughput.

\section{B. Analysis of BER:}

In a communication system, the receiver side BER may be affected by transmission channel noise, interference, distortion, bit synchronization problems, attenuation, The BER may be improved by choosing a strong signal strength (unless this causes cross-talk and more bit errors), by choosing a slow and robust modulation scheme or line codingscheme, and by applying channel coding schemes such as redundant forward error correction codes.

$$
\mathrm{BER}=\frac{1}{2} \sqrt{E_{\text {b }} / N_{0}}
$$

In optical communication, $\mathrm{BER}(\mathrm{dB})$ vs. Received Power $(\mathrm{dBm})$ is usually used; while in wireless communication, $\mathrm{BER}(\mathrm{dB})$ vs. $\mathrm{SNR}(\mathrm{dB})$ is used.Measuring the bit error ratio helps people choose the appropriate forward error correction codes. Since most such codes correct only bit-flips, but not bit-insertions or bit-deletions, the Hamming distance metric is the appropriate way to measure the number of bit errors. Many FEC coders also continuously measure the current BER.

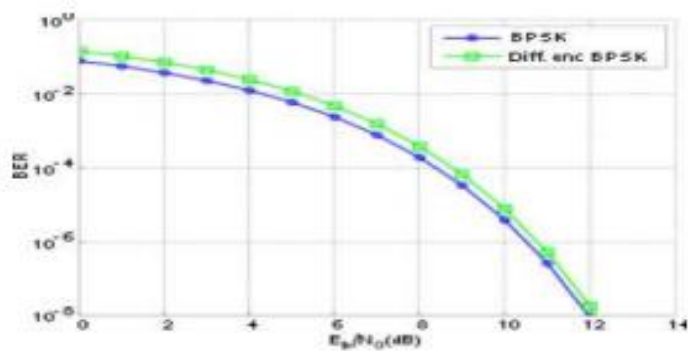


FIGURE 14: BER comparison between BPSK and differentially encoded BPSK with gray coding technique

\section{The Bit Error Rate (BER) Performance in} Multi-Carrier (OFDM) And Single-Carrier:

The spectacular growth of wireless communication tools has escalated the number of mobile subscribers from almost 700 million in 2000 to more than 4 billion in 2009. The huge number of subscribers has led to several issues with how service is provided. The high user demand has forced developers to overcome the problems of the old analog systems and to introduce OFDM as a promising technique that can fulfill users' high demands. This technique matches well with high data rate connection and provides a higher capacity for the subscribers' usage.The OFDM, as a multi-carrier, is more complex than the single-carrier transmission scheme. However, the OFDM technique maintains better performance for high data rate in terms of bit error rate (BER). In this thesis a comparison has been presented between the multi-carrier OFDM and the single-carrier to prove, in a simulation form, the theoretical point of view. Despite the advantages of using the OFDM scheme, there are several drawbacks. One of these negatives is the high peak to average power ratio (PAPR).

To overcome this problem, there are power reduction techniques that can be applied to the signal to reduce the high power. One of these techniques is the clipping and filtering technique. A maximum level is sited for the transmitted signal to reduce the power and afterward, the signal goes through a filter to remove the influence of the inband distortion and out-of-band radiation.

\section{Manual Calculations}

The BER is the likelihood of a bit misinterpretation due to electrical noise . Considering a bipolar NRZ transmission, we have $x_{1}(t)=A+w(t)$ for $a^{\prime \prime} 1 "$ and $x_{2}(t)=-A+$ $\mathbf{w}(\mathbf{t})$ for a "0". Each of $x_{1}(t)$ and $x_{2}(t)$ has a period of T. Knowing that the noise has a bilateral spectral

density $\mathrm{N0/2}$

$$
P_{g}=0.5 \mathrm{erfc} \sqrt{E_{b} / N_{0}} \pm \S
$$

\section{BER SIMULATION RESULTS}

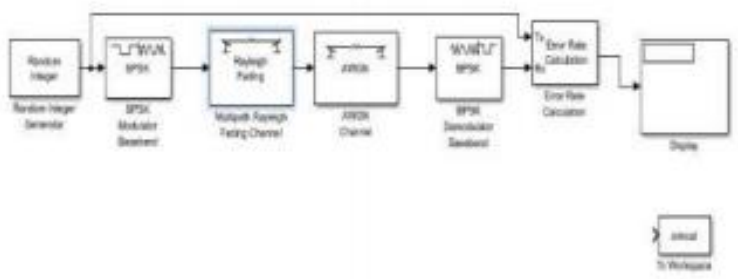

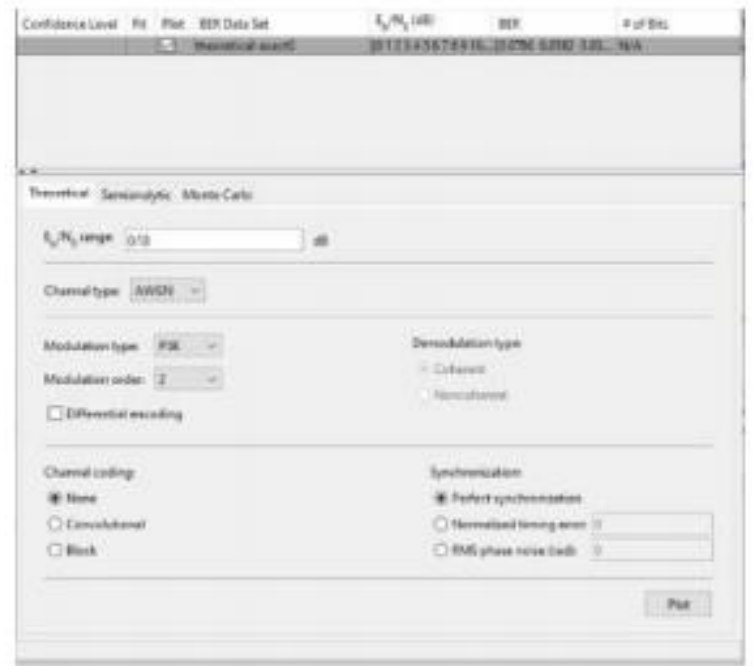

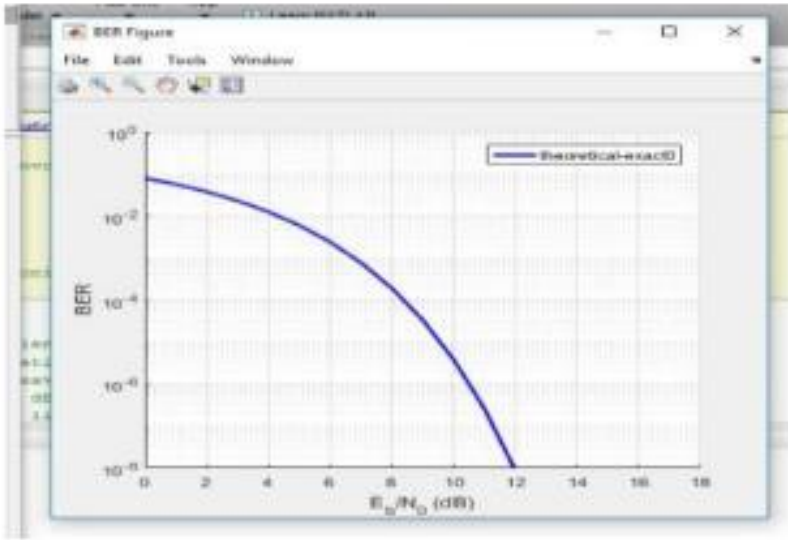

FIGURE 16:BER vs SNR

\section{A. $B P S K$}

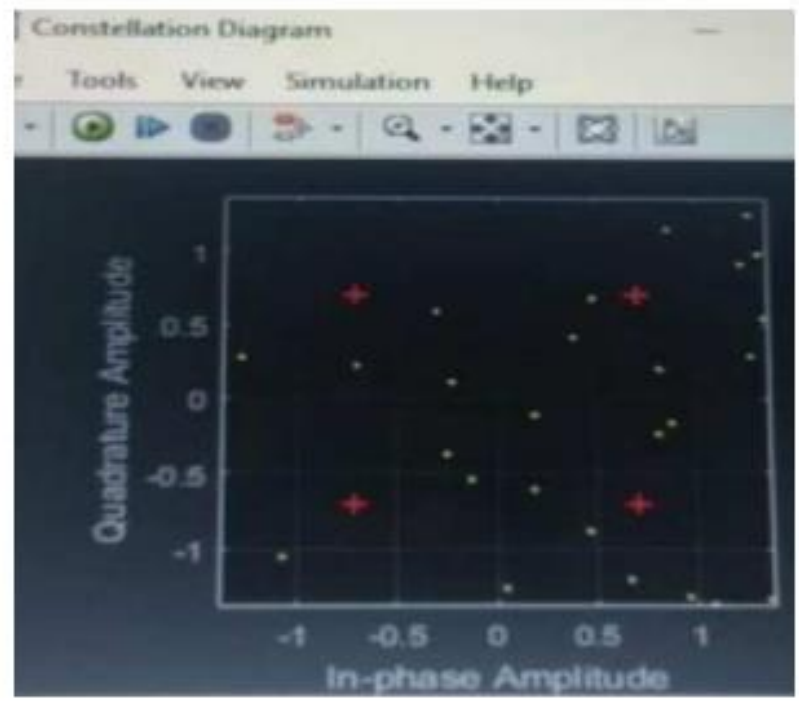

FIGURE 15: Block Diagram 


\section{B. $Q P S K$}

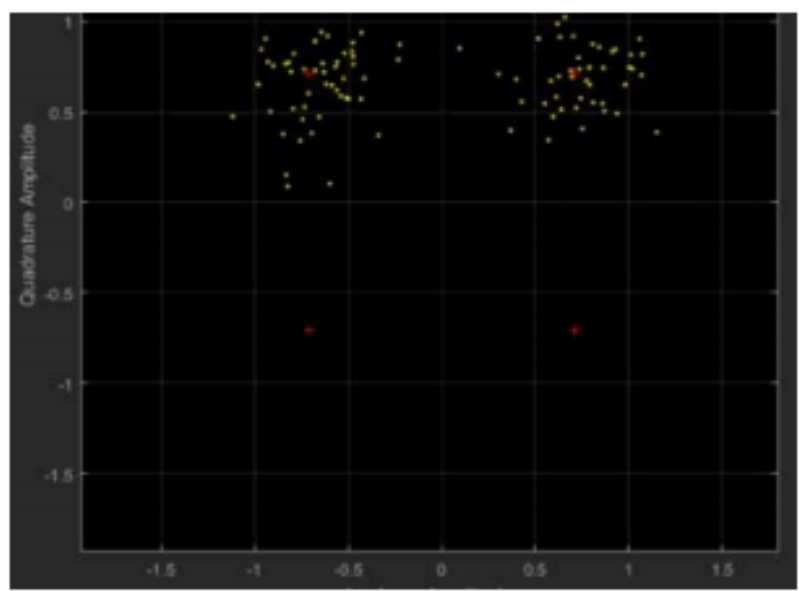

\section{C. $16 Q A M$}

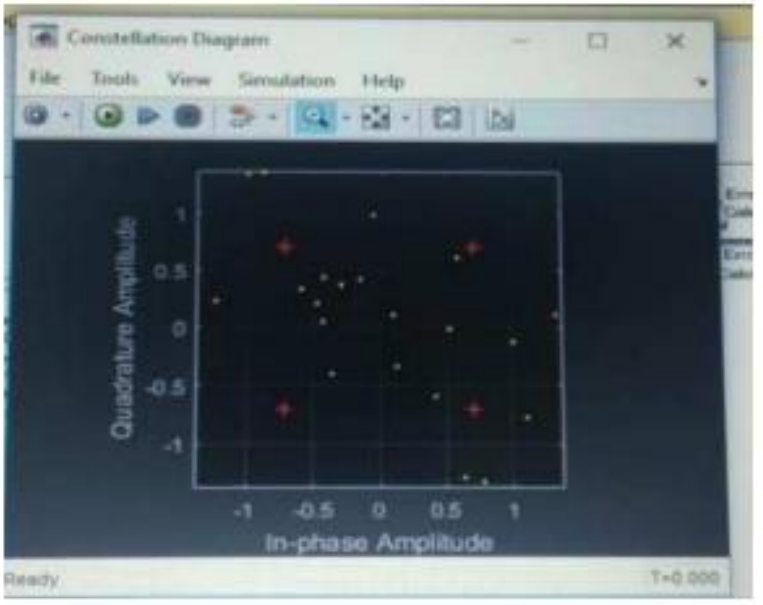

\section{CONCLUSION}

In this report the formal assembling of OFDM block with input random bit stream that is coded into LDPC is given to observe the flow of the data through the AWGN channel and received data is checked for good amount of quality with less errors. Signal to noise ratio and Bit error rates were observed in this OFDM system where some comparison of 8 QPSK and 16 QPSK and QAM modulated signals were used. Multi carrier MIMO is often used for $5 \mathrm{G}$ technologies to achieve faster data rates upto 1GBPS. This work will enhance the knowledge of the $5 \mathrm{G}$ enthusiasts to explore more possibilities of techniques to be incorporated in the project.

\section{FUTURE SCOPE}

It is undoubtedly known that the data speeds are directly impacting the growth of any economy in $21^{\text {st }}$ century.

On this note, $5 \mathrm{G}$ wireless communications would bring the life more easy and fast in processing speeds of computations through rapid pace in integrated electronics and millimeter wave technologies together. It is indeed accepted by many research communities that the data transmission technologies will surface the lifestyles very soon with growing interest of sharing the ideas in no time irrespective of time, place \& power. Industry 4.0, Internet of Things (IoT), Internet of
Everything (IoE), Terra hertz technologies, Industrial Automation through Artificial Intelligence, Data Science, Data Analytics, Data Mining, Cyber security, Space Colonization, Rocket Science, Satellite Technology, Remote Sensing, Nano-Robotics, Remote Medicine, Intelligent Genetics etc, are some of the most promising technologies for the future century which is only possible with the ease in data transmission that was emphasized in this report.

\section{REFERENCES}

[1] Comprehensive Analysis of UFMC with OFDM and FBMC, K. Krishna Kishore, P. Rajesh Umar, V. Jagan Naveen .,

[2] 5G Waveform Candidates by Rohde \& Schwarz.,

[3] Peak to Average Power Ratio (PAPR) reduction in OFDM for a WLAN network using SLM technique, ISBN: 978-1-4244-8679-3, IEEE.

Vemakoti Vivekanand M.Tech, MBA, is a curious researcher in quantum electrodynamics and high speed signal transmissions though had a deep insights on photovoltaic cells' performance calibrations in his previous research papers. He has 6 years of experience in teaching at various engineering and academic institutes, presently working as IIT - Physics lecturer in a national level schooling institute. He is ready to collaborate any level of research stimulus in the aforesaid domains or any promising interdisciplinary prospects of science and technology.

Sathish Kumar Yegireddi M.Tech (Embedded System) Currently working in Department of ECE,IIIT Srikakulam, RAJIV GANDH UNIVERSITY OF KNOWLEDGE TECHNOLOGIES(RGUKT),Andhra Pradesh, India; sathishkumaryegireddi@gmail.com, 